\title{
Subclass of univalent harmonic functions defined by dual convolution
}

\section{Rabha M El-Ashwah*}

\section{"Correspondence:}

r_elashwah@yahoo.com

Department of Mathematics,

Faculty of Science, Damietta

University, New Damietta, 34517,

Egypt

\begin{abstract}
In the present paper, we study a subclass of univalent harmonic functions defined by convolution and integral convolution. We obtain the basic properties such as coefficient characterization and distortion theorem, extreme points and convolution condition.
\end{abstract}

MSC: $30 C 45 ; 30 C 50$

Keywords: harmonic function; univalent; sense-preserving; integral convolution

\section{Introduction}

A continuous function $f=u+i v$ is a complex-valued harmonic function in a simply connected complex domain $D \subset \mathbb{C}$ if both $u$ and $v$ are real harmonic in $D$. It was shown by Clunie and Sheil-Small [1] that such a harmonic function can be represented by $f=h+\bar{g}$, where $h$ and $g$ are analytic in $D$. Also, a necessary and sufficient condition for $f$ to be locally univalent and sense-preserving in $D$ is that $\left|h^{\prime}(z)\right|>\left|g^{\prime}(z)\right|$ (see also [2-4] and [5]).

Denote by $S_{H}$ the class of functions $f$ that are harmonic univalent and sense-preserving in the open unit disc $U=\{z \in \mathbb{C}:|z|<1\}$, for which $f(0)=h(0)=f_{z}^{\prime}(0)-1=0$. Then for $f=h+\bar{g} \in S_{H}$ we may express the analytic functions $h$ and $g$ as

$$
h(z)=z+\sum_{n=2}^{\infty} a_{n} z^{n}, \quad g(z)=\sum_{n=1}^{\infty} b_{n} z^{n}, \quad\left|b_{1}\right|<1
$$

Clunie and Sheil-Small [1] investigated the class $S_{H}$ as well as its geometric subclasses and obtained some coefficient bounds.

Also, let $S_{\bar{H}}$ denote the subclass of $S_{H}$ consisting of functions $f=h+\bar{g}$ such that the functions $h$ and $g$ are of the form

$$
h(z)=z-\sum_{n=2}^{\infty}\left|a_{n}\right| z^{n}, \quad g(z)=\sum_{n=1}^{\infty}\left|b_{n}\right| z^{n}, \quad\left|b_{1}\right|<1 .
$$

Recently Kanas and Wisniowska [6] (see also Kanas and Srivastava [7]) studied the class of $k$-uniformly convex analytic functions, denoted by $k-U C V, k \geq 0$, so that $\phi \in k-U C V$ if and only if

$$
\operatorname{Re}\left\{1+\frac{(z-\zeta) \phi^{\prime \prime}(z)}{\phi^{\prime}(z)}\right\} \geq 0 \quad(|\zeta| \leq k ; z \in U) .
$$


For $\theta \in \mathbb{R}$, if we let $\zeta=-k z e^{i \theta}$, then condition (1.3) can be written as

$$
\operatorname{Re}\left\{1+\left(1+k e^{i \theta}\right) \frac{z \phi^{\prime \prime}(z)}{\phi^{\prime}(z)}\right\} \geq 0
$$

Kim et al. [8] introduced and studied the class $H C V(k, \alpha)$ consisting of functions $f=h+\bar{g}$, such that $h$ and $g$ are given by (1.1), and satisfying the condition

$$
\operatorname{Re}\left\{1+\left(1+k e^{i \theta}\right) \frac{z^{2} h^{\prime \prime}(z)+\overline{2 z g^{\prime}(z)+z^{2} g^{\prime \prime}(z)}}{z h^{\prime}(z)-\overline{z g^{\prime}(z)}}\right\} \geq \alpha \quad(0 \leq \alpha<1 ; \theta \in \mathbb{R} ; k \geq 0)
$$

Also, the class of $k-U S T$ uniformly starlike functions is defined by using (1.4) as the class of all functions $\psi(z)=z \phi^{\prime}(z)$ such that $\phi \in k-U C V$, then $\psi(z) \in k-U S T$ if and only if

$$
\operatorname{Re}\left\{\left(1+k e^{i \theta}\right) \frac{z \psi^{\prime}(z)}{\psi(z)}-k e^{i \theta}\right\} \geq 0
$$

Generalizing the class $k-U S T$ to include harmonic functions, we let $H S T(k, \alpha)$ denote the class of functions $f=h+\bar{g}$, such that $h$ and $g$ are given by (1.1), which satisfies the condition

$$
\operatorname{Re}\left\{\left(1+k e^{i \theta}\right) \frac{z f^{\prime}(z)}{z^{\prime} f(z)}-k e^{i \theta}\right\} \geq \alpha \quad(0 \leq \alpha<1 ; \theta \in \mathbb{R} ; k \geq 0)
$$

Replacing $h+\bar{g}$ for $f$ in (1.7), we have

$$
\operatorname{Re}\left\{\left(1+k e^{i \theta}\right) \frac{z h^{\prime}(z)-\overline{z g^{\prime}(z)}}{h(z)+\overline{g(z)}}-k e^{i \theta}\right\} \geq \alpha \quad(0 \leq \alpha<1 ; \theta \in \mathbb{R} ; k \geq 0) .
$$

The convolution of two functions of the form

$$
f(z)=z+\sum_{n=2}^{\infty} a_{n} z^{n} \quad \text { and } \quad F(z)=z+\sum_{n=2}^{\infty} A_{n} z^{n}
$$

is defined as

$$
(f * F)(z)=z+\sum_{n=2}^{\infty} a_{n} A_{n} z^{n}
$$

while the integral convolution is defined by

$$
(f \diamond F)(z)=z+\sum_{n=2}^{\infty} \frac{a_{n} A_{n}}{n} z^{n} .
$$

From (1.9) and (1.10), we have

$$
(f \diamond F)(z)=\int_{0}^{z} \frac{(f * F)(t)}{t} d t .
$$


Now we consider the subclass $\operatorname{HST}(\phi, \psi, k, \alpha)$ consisting of functions $f=h+\bar{g}$, such that $h$ and $g$ are given by (1.1), and satisfying the condition

$$
\operatorname{Re}\left\{\left(1+k e^{i \theta}\right) \frac{h(z) * \varphi(z)-\overline{g(z) * \chi(z)}}{h(z) \diamond \varphi(z)+\overline{g(z) \diamond \chi(z)}}-k e^{i \theta}\right\} \geq \alpha \quad(0 \leq \alpha<1 ; k \geq 0 ; \theta \text { real })
$$

where

$$
\varphi(z)=z+\sum_{n=2}^{\infty} \lambda_{n} z^{n} \quad\left(\lambda_{n} \geq 0\right) \quad \text { and } \quad \chi(z)=z+\sum_{n=2}^{\infty} \mu_{n} z^{n} \quad\left(\mu_{n} \geq 0\right) .
$$

We further consider the subclass $\overline{H S T}(\phi, \chi, k, \alpha)$ of $\operatorname{HST}(\phi, \chi, k, \alpha)$ for $h$ and $g$ given by (1.2).

We note that

(i) $\overline{H S T}(\phi, \chi, 0, \alpha)=\overline{H S}(\phi, \chi, \alpha)$ (see Dixit et al. [9]);

(ii) $\overline{H S T}\left(\frac{z}{(1-z)^{2}}, \frac{z}{(1-z)^{2}}, 1, \alpha\right)=G_{\bar{H}}(\alpha)$ (see Rosy et al. [10]);

(iii) $\overline{H S T}\left(\frac{z+z^{2}}{(1-z)^{3}}, \frac{z+z^{2}}{(1-z)^{3}}, k, \alpha\right)=\bar{H} C V(k, \alpha)$ (see Kim et al. [8]);

(iv) $\overline{H S T}\left(\frac{z}{(1-z)^{2}}, \frac{z}{(1-z)^{2}}, 0, \alpha\right)=T_{H}^{*}(\alpha)$ (see Jahangiri [3], see also Joshi and Darus [11]);

(v) $\overline{H S T}\left(\frac{z+z^{2}}{(1-z)^{3}}, \frac{z+z^{2}}{(1-z)^{3}}, 0, \alpha\right)=C_{H}(\alpha)$ (see Jahangiri [3], see also Joshi and Darus [11]).

In this paper, we extend the results of the above classes to the classes $H S T(\phi, \chi, k, \alpha)$ and $\overline{H S T}(\phi, \chi, k, \alpha)$, we also obtain some basic properties for the class $\overline{H S T}(\phi, \chi, k, \alpha)$.

\section{Coefficient characterization and distortion theorem}

Unless otherwise mentioned, we assume throughout this paper that $\varphi(z)$ and $\chi(z)$ are given by (1.12), $0 \leq \alpha<1, k \geq 0$ and $\theta$ is real. We begin with a sufficient condition for functions in the class $\operatorname{HST}(\phi, \chi, k, \alpha)$.

Theorem 1 Let $f=h+\bar{g}$ be such that $h$ and $g$ are given by (1.1). Furthermore, let

$$
\sum_{n=2}^{\infty} \frac{\lambda_{n}}{n}\left(\frac{(1+k) n-(k+\alpha)}{(1-\alpha)}\right)\left|a_{n}\right|+\sum_{n=1}^{\infty} \frac{\mu_{n}}{n}\left(\frac{(1+k) n+(k+\alpha)}{(1-\alpha)}\right)\left|b_{n}\right| \leq 1,
$$

where

$$
\begin{aligned}
& n^{2}(1-\alpha) \leq \lambda_{n}[(1+k) n-(k+\alpha)] \text { and } n^{2}(1-\alpha) \leq \mu_{n}[(1+k) n+(k+\alpha)] \\
& \quad \text { for } n \geq 2
\end{aligned}
$$

Then $f$ is sense-preserving, harmonic univalent in $U$ and $f \in H S T(\phi, \chi, k, \alpha)$.

Proof First we note that $f$ is locally univalent and sense-preserving in $U$. This is because

$$
\begin{aligned}
\left|h^{\prime}(z)\right| & \geq 1-\sum_{n=2}^{\infty} n\left|a_{n}\right| r^{n-1}>1-\sum_{n=2}^{\infty} n\left|a_{n}\right| \geq 1-\sum_{n=2}^{\infty} \frac{\lambda_{n}}{n}\left(\frac{(1+k) n-(k+\alpha)}{(1-\alpha)}\right)\left|a_{n}\right| \\
& \geq \sum_{n=1}^{\infty} \frac{\mu_{n}}{n}\left(\frac{(1+k) n+(k+\alpha)}{(1-\alpha)}\right)\left|b_{n}\right| \geq \sum_{n=1}^{\infty} n\left|b_{n}\right| \geq \sum_{n=1}^{\infty} n\left|b_{n}\right| r^{k-1}>\left|g^{\prime}(z)\right| .
\end{aligned}
$$


To show that $f$ is univalent in $U$, suppose $z_{1}, z_{2} \in U$ so that $z_{1} \neq z_{2}$, then

$$
\begin{aligned}
\left|\frac{f\left(z_{1}\right)-f\left(z_{2}\right)}{h\left(z_{1}\right)-h\left(z_{2}\right)}\right| & \geq 1-\left|\frac{g\left(z_{1}\right)-g\left(z_{2}\right)}{h\left(z_{1}\right)-h\left(z_{2}\right)}\right|=1-\left|\frac{\sum_{n=1}^{\infty} b_{n}\left(z_{1}^{n}-z_{2}^{n}\right)}{\left(z_{1}-z_{2}\right)+\sum_{n=2}^{\infty} a_{n}\left(z_{1}^{n}-z_{2}^{n}\right)}\right| \\
& \geq 1-\frac{\sum_{n=1}^{\infty} n\left|b_{n}\right|}{1-\sum_{n=2}^{\infty} n\left|a_{n}\right|}>1-\frac{\sum_{n=1}^{\infty} \frac{\mu_{n}}{n}\left(\frac{(1+k) n+(k+\alpha)}{(1-\alpha)}\right)\left|b_{n}\right|}{1-\sum_{n=2}^{\infty} \frac{\lambda_{n}}{n}\left(\frac{(1+k) n-(k+\alpha)}{(1-\alpha)}\right)\left|a_{n}\right|} \geq 0 .
\end{aligned}
$$

Now, we prove that $f \in H S T(\phi, \psi, k, \alpha)$, by definition, we only need to show that if (2.1) holds, then condition (1.11) is satisfied. From (1.11), it suffices to show that

$$
\operatorname{Re}\left\{\frac{\left(1+k e^{i \theta}\right)(h(z) * \varphi(z)-\overline{g(z) * \chi(z)})-\left(k e^{i \theta}+\alpha\right)(h(z) \diamond \varphi(z)+\overline{g(z) \diamond \chi(z)})}{h(z) \diamond \varphi(z)+\overline{g(z) \diamond \chi(z)}}\right\}
$$

$\geq 0$.

Substituting for $h, g, \varphi$ and $\chi$ in (2.2) and dividing by $(1-\alpha) z$, we obtain $\operatorname{Re} \frac{A(z)}{B(z)} \geq 0$, where

$$
\begin{aligned}
A(z)= & +\sum_{n=2}^{\infty} \frac{\lambda_{n}}{n} \frac{\left(1+k e^{i \theta}\right) n-\left(k e^{i \theta}+\alpha\right)}{(1-\alpha)} a_{n} z^{n-1} \\
& -\left(\frac{\bar{z}}{z}\right) \sum_{n=1}^{\infty} \frac{\mu_{n}}{n} \frac{\left(1+k e^{i \theta}\right) n+\left(k e^{i \theta}+\alpha\right)}{(1-\alpha)} b_{n} \bar{z}^{n-1}
\end{aligned}
$$

and

$$
B(z)=1+\sum_{n=2}^{\infty} \frac{\lambda_{n}}{n} a_{n} z^{n-1}+\left(\frac{\bar{z}}{z}\right) \sum_{n=1}^{\infty} \frac{\mu_{n}}{n} b_{n} \bar{z}^{n-1}
$$

Using the fact that $\operatorname{Re}(w) \geq 0$ if and only if $|1+w| \geq|1-w|$ in $U$, it suffices to show that $|A(z)+B(z)|-|A(z)-B(z)| \geq 0$. Substituting for $A(z)$ and $B(z)$ gives

$$
\begin{aligned}
\mid A(z) & +B(z)|-| A(z)-B(z) \mid \\
= & \mid 2+\sum_{n=2}^{\infty} \frac{\lambda_{n}}{n} \frac{\left(1+k e^{i \theta}\right) n-\left(k e^{i \theta}+2 \alpha-1\right)}{(1-\alpha)} a_{n} z^{n-1} \\
& -\left(\frac{\bar{z}}{z}\right) \sum_{n=1}^{\infty} \frac{\mu_{n}}{n} \frac{\left(1+k e^{i \theta}\right) n+\left(k e^{i \theta}+2 \alpha-1\right)}{(1-\alpha)} b_{n} \bar{z}^{n-1} \mid \\
& -\mid \sum_{n=2}^{\infty} \frac{\lambda_{n}}{n} \frac{\left(1+k e^{i \theta}\right) n-\left(1+k e^{i \theta}\right)}{(1-\alpha)} a_{n} z^{n-1} \\
& -\left(\frac{\bar{z}}{z}\right) \sum_{n=1}^{\infty} \frac{\mu_{n}}{n} \frac{\left(1+k e^{i \theta}\right) n+\left(1+k e^{i \theta}\right)}{(1-\alpha)} b_{n} \bar{z}^{n-1} \mid \\
\geq 2 & -\sum_{n=2}^{\infty} \frac{\lambda_{n}}{n} \frac{(1+k) n-(k+2 \alpha-1)}{(1-\alpha)}\left|a_{n}\right||z|^{n-1} \\
& -\sum_{n=1}^{\infty} \frac{\mu_{n}}{n} \frac{(1+k) n+(k+2 \alpha-1)}{(1-\alpha)}\left|b_{n}\right||z|^{n-1}
\end{aligned}
$$




$$
\begin{aligned}
& -\sum_{n=2}^{\infty} \frac{\lambda_{n}}{n} \frac{(1+k) n-(1+k)}{(1-\alpha)}\left|a_{n+1}\right||z|^{n-1} \\
& -\sum_{n=1}^{\infty} \frac{\mu_{n}}{n} \frac{(1+k) n+(1+k)}{(1-\alpha)}\left|b_{n}\right||z|^{n-1} \\
\geq & 2\left\{1-\sum_{n=2}^{\infty} \frac{\lambda_{n}}{n} \frac{(1+k) n-(k+\alpha)}{(1-\alpha)}\left|a_{n}\right|-\sum_{n=1}^{\infty} \frac{\mu_{n}}{n} \frac{(1+k) n+(k+\alpha)}{(1-\alpha)}\left|b_{n}\right|\right\}
\end{aligned}
$$$$
\geq 0 \text { by }(2.1) \text {. }
$$

The harmonic functions

$$
\begin{aligned}
f(z)= & z+\sum_{n=2}^{\infty} \frac{n}{\lambda_{n}} \frac{(1-\alpha)}{(1+k) n-(k+\alpha)} x_{n} z^{n} \\
& +\sum_{n=1}^{\infty} \frac{n}{\mu_{n}} \frac{(1-\alpha)}{(1+k) n+(k+\alpha)} \bar{y}_{n} \bar{z}^{n},
\end{aligned}
$$

where $\sum_{n=2}^{\infty}\left|x_{n}\right|+\sum_{n=1}^{\infty}\left|y_{n}\right|=1$, show that the coefficient bound given by (2.1) is sharp. The functions of the form (2.3) are in the class $\operatorname{HST}(\phi, \chi, k, \alpha)$ because

$$
\begin{aligned}
& \sum_{n=2}^{\infty}\left[\frac{\lambda_{n}}{n} \frac{(1+k) n-(k+\alpha)}{(1-\alpha)}\left|a_{n}\right|+\sum_{n=1}^{\infty} \frac{\mu_{n}}{n} \frac{(1+k) n+(k+\alpha)}{(1-\alpha)}\left|b_{n}\right|\right] \\
& \quad=\sum_{n=2}^{\infty}\left|x_{n}\right|+\sum_{n=1}^{\infty}\left|y_{n}\right|=1 .
\end{aligned}
$$

This completes the proof of Theorem 1 .

In the following theorem, it is shown that condition (2.1) is also necessary for functions $f=h+\bar{g}$, where $h$ and $g$ are given by (1.2).

Theorem 2 Let $f=h+\bar{g}$ be such that $h$ and $g$ are given by (1.2). Then $f \in \overline{H S T}(\phi, \chi, k, \alpha)$ if and only if

$$
\sum_{n=2}^{\infty} \frac{\lambda_{n}}{n}\left(\frac{(1+k) n-(k+\alpha)}{(1-\alpha)}\right)\left|a_{n}\right|+\sum_{n=1}^{\infty} \frac{\mu_{n}}{n}\left(\frac{(1+k) n+(k+\alpha)}{(1-\alpha)}\right)\left|b_{n}\right| \leq 1
$$

Proof Since $\overline{H S T}(\phi, \chi, k, \alpha) \subset H S T(\phi, \chi, k, \alpha)$, we only need to prove the 'only if' part of the theorem. To this end, we notice that the necessary and sufficient condition for $f \in$ $\overline{H S T}(\phi, \chi, k, \alpha)$ is that

$$
\operatorname{Re}\left\{\left(1+k e^{i \theta}\right) \frac{h(z) * \varphi(z)-\overline{g(z) * \chi(z)}}{h(z) \diamond \varphi(z)+\overline{g(z) \diamond \chi(z)}}-k e^{i \theta}\right\} \geq \alpha .
$$

This is equivalent to

$$
\operatorname{Re}\left\{\frac{\left(1+k e^{i \theta}\right)(h(z) * \varphi(z)-\overline{g(z) * \chi(z)})-\left(k e^{i \theta}+\alpha\right)(h(z) \diamond \varphi(z)+\overline{g(z) \diamond \chi(z)})}{h(z) \diamond \varphi(z)+\overline{g(z) \diamond \chi(z)}}\right\}>0,
$$


which implies that

$$
\begin{aligned}
\operatorname{Re}\{ & \frac{(1-\alpha) z-\sum_{n=2}^{\infty} \frac{\lambda_{n}}{n}\left[\left(1+k e^{i \theta}\right) n-\left(k e^{i \theta}+\alpha\right)\right]\left|a_{n}\right| z^{n}}{z-\sum_{n=2}^{\infty} \frac{\lambda_{n}}{n}\left|a_{n}\right| z^{n}+\sum_{n=1}^{\infty} \frac{\mu_{n}}{n}\left|b_{n}\right| \bar{z}^{n}} \\
& \left.-\frac{\sum_{n=1}^{\infty} \frac{\mu_{n}}{n}\left[\left(1+k e^{i \theta}\right) n+\left(k e^{i \theta}+\alpha\right)\right]\left|b_{n}\right| \bar{z}^{n}}{z-\sum_{n=2}^{\infty} \frac{\lambda_{n}}{n}\left|a_{n}\right| z^{n}+\sum_{n=1}^{\infty} \frac{\mu_{n}}{n}\left|b_{n}\right| \bar{z}^{n}}\right\} \\
= & \operatorname{Re}\left\{\frac{(1-\alpha)-\sum_{n=2}^{\infty} \frac{\lambda_{n}}{n}\left[\left(1+k e^{i \theta}\right) n-\left(k e^{i \theta}+\alpha\right)\right]\left|a_{n}\right| z^{n-1}}{1-\sum_{n=2}^{\infty} \frac{\lambda_{n}}{n}\left|a_{n}\right| z^{n-1}+\left(\frac{\bar{z}}{z}\right) \sum_{n=1}^{\infty} \frac{\mu_{n}}{n}\left|b_{n}\right| \bar{z}^{n-1}}\right. \\
& \left.-\frac{\left(\frac{\bar{z}}{z}\right) \sum_{n=1}^{\infty} \frac{\mu_{n}}{n}\left[\left(1+k e^{i \theta}\right) n+\left(k e^{i \theta}+\alpha\right)\right]\left|b_{n}\right| \bar{z}^{n-1}}{1-\sum_{n=2}^{\infty} \frac{\lambda_{n}}{n}\left|a_{n}\right| z^{n}+\left(\frac{\bar{z}}{z}\right) \sum_{n=1}^{\infty} \frac{\mu_{n}}{n}\left|b_{n}\right| \bar{z}^{n-1}}\right\}>0,
\end{aligned}
$$

since $\operatorname{Re}\left(e^{i \theta}\right) \leq\left|e^{i \theta}\right|=1$, the required condition (2.5) is equivalent to

$$
\begin{aligned}
& \left\{\frac{1-\sum_{n=2}^{\infty} \frac{\lambda_{n}}{n} \frac{(1+k) n-(k+\alpha)}{(1-\alpha)}\left|a_{n}\right| r^{n-1}}{1-\sum_{n=2}^{\infty} \frac{\lambda_{n}}{n}\left|a_{n}\right| r^{n-1}+\sum_{n=1}^{\infty} \frac{\mu_{n}}{n}\left|b_{n}\right| r^{n-1}}-\frac{\sum_{n=1}^{\infty} \frac{\mu_{n}}{n} \frac{(1+k) n+(k+\alpha)}{(1-\alpha)}\left|b_{n}\right| r^{n-1}}{1-\sum_{n=2}^{\infty} \frac{\lambda_{n}}{n}\left|a_{n}\right| r^{n-1}+\sum_{n=1}^{\infty} \frac{\mu_{n}}{n}\left|b_{n}\right| r^{n-1}}\right\} \\
& \quad \geq 0 .
\end{aligned}
$$

If condition (2.4) does not hold, then the numerator in (2.6) is negative for $z=r$ sufficiently close to 1 . Hence there exists $z_{0}=r_{0}$ in $(0,1)$ for which the quotient in $(2.6)$ is negative. This contradicts the required condition for $f \in \overline{H S T}(\phi, \chi, k, \alpha)$, and so the proof of Theorem 2 is completed.

Theorem 3 Let $f \in \overline{H S T}(\phi, \chi, k, \alpha)$. Then, for $|z|=r<1,\left|b_{1}\right|<\frac{1-\alpha}{2 k+\alpha+1}$ and

$$
D_{n} \leq \frac{\lambda_{n}}{n}, \quad E_{n} \leq \frac{\mu_{n}}{n} \quad \text { for } n \geq 2 \quad \text { and } \quad C=\min \left\{D_{2}, E_{2}\right\}
$$

we have

$$
|f(z)| \leq\left(1+\left|b_{1}\right|\right) r+\left\{\frac{(1-\alpha)}{C(2+k-\alpha)}-\frac{2 k+1+\alpha}{C(2+k-\alpha)}\left|b_{1}\right|\right\} r^{2}
$$

and

$$
|f(z)| \geq\left(1-\left|b_{1}\right|\right) r-\left\{\frac{(1-\alpha)}{C(2+k-\alpha)}-\frac{2 k+1+\alpha}{C(2+k-\alpha)}\left|b_{1}\right|\right\} r^{2} .
$$

The results are sharp.

Proof We prove the left-hand side inequality for $|f|$. The proof for the right-hand side inequality can be done by using similar arguments.

Let $f \in \overline{H S T}(\phi, \chi, k, \alpha)$, then we have

$$
\begin{aligned}
|f(z)| & =\left|z-\sum_{n=2}^{\infty}\right| a_{n}\left|z^{n}+\sum_{n=1}^{\infty}\right| b_{n}\left|\bar{z}^{n}\right| \\
& \geq r-\left|b_{1}\right| r-\sum_{n=2}^{\infty}\left(\left|a_{n}\right|+\left|b_{n}\right|\right) r^{2}
\end{aligned}
$$




$$
\begin{aligned}
\geq & r-\left|b_{1}\right| r \\
& -\frac{(1-\alpha)}{C(2+k-\alpha)} \sum_{n=2}^{\infty} \frac{C((1+k) n-(k+\alpha))}{(1-\alpha)}\left(\left|a_{n}\right|+\left|b_{n}\right|\right) r^{2} \\
\geq & r-\left|b_{1}\right| r \\
& -\frac{(1-\alpha)}{C(2+k-\alpha)} \sum_{n=2}^{\infty}\left\{\frac{C((1+k) n-(k+\alpha))}{(1-\alpha)}\left|a_{n}\right|\right. \\
& \left.+\frac{C((1+k) n+(k+\alpha))}{(1-\alpha)}\left|b_{n}\right|\right\} r^{2} \\
\geq & \left(1-\left|b_{1}\right|\right) r-\frac{(1-\alpha)}{C(2+k-\alpha)}\left\{1-\frac{2 k+1+\alpha}{(1-\alpha)}\left|b_{1}\right|\right\} r^{2} \\
\geq & \left(1-\left|b_{1}\right|\right) r-\left\{\frac{(1-\alpha)}{C(2+k-\alpha)}-\frac{2 k+1+\alpha}{C(2+k-\alpha)}\left|b_{1}\right|\right\} r^{2} .
\end{aligned}
$$

The bounds given in Theorem 3 are respectively attained for the following functions:

$$
f(z)=z+\left|b_{1}\right| \bar{z}+\left(\frac{(1-\alpha)}{C(2+k-\alpha)}-\frac{2 k+1+\alpha}{C(2+k-\alpha)}\left|b_{1}\right|\right) \bar{z}^{2}
$$

and

$$
f(z)=\left(1-\left|b_{1}\right|\right) z-\left(\frac{(1-\alpha)}{C(2+k-\alpha)}-\frac{2 k+1+\alpha}{C(2+k-\alpha)}\left|b_{1}\right|\right) z^{2}
$$

The following covering result follows from the left side inequality in Theorem 3.

Corollary 1 Let $f \in \overline{H S T}(\phi, \chi, k, \alpha)$, then for $\left|b_{1}\right|<\frac{1-\alpha}{2 k+\alpha+1}$ the set

$$
\left\{w:|w|<1-\frac{(1-\alpha)}{C(2+k-\alpha)}-\left(1-\frac{2 k+1+\alpha}{C(2+k-\alpha)}\right)\left|b_{1}\right|\right\}
$$

is included in $f(U)$, where $C$ is given by (2.7).

\section{Extreme points}

Our next theorem is on the extreme points of convex hulls of the class $\overline{\operatorname{HST}}(\phi, \chi, k, \alpha)$, denoted by $\operatorname{clco} \overline{H S T}(\phi, \chi, k, \alpha)$.

Theorem 4 Let $f=h+\bar{g}$ be such that $h$ and $g$ are given by (1.2). Then $f \in \operatorname{clco} \overline{H S T}(\phi, \chi, k, \alpha)$ if and only iff can be expressed as

$$
f(z)=\sum_{n=1}^{\infty}\left[X_{n} h_{n}(z)+Y_{n} g_{n}(z)\right]
$$

where

$$
\begin{aligned}
& h_{1}(z)=z, \\
& h_{n}(z)=z-\frac{n(1-\alpha)}{\lambda_{n}((1+k) n-(k+\alpha))} z^{n} \quad(n \geq 2),
\end{aligned}
$$




$$
\begin{aligned}
& g_{n}(z)=z+\frac{n(1-\alpha)}{\mu_{n}((1+k) n+(k+\alpha))} \bar{z}^{n} \quad(n \geq 1), \\
& X_{n} \geq 0, \quad Y_{n} \geq 0, \quad \sum_{n=1}^{\infty}\left[X_{n}+Y_{n}\right]=1 .
\end{aligned}
$$

In particular, the extreme points of the class $\overline{\operatorname{HST}}(\phi, \chi, k, \alpha)$ are $\left\{h_{n}\right\}$ and $\left\{g_{n}\right\}$, respectively.

Proof For functions $f(z)$ of the form (3.1), we have

$$
f(z)=\sum_{n=1}^{\infty}\left[X_{n}+Y_{n}\right] z-\sum_{n=2}^{\infty} \frac{n(1-\alpha)}{\lambda_{n}((1+k) n-(k+\alpha))} X_{n} z^{n}+\sum_{n=1}^{\infty} \frac{n(1-\alpha)}{\mu_{n}((1+k) n+(k+\alpha))} Y_{n} \bar{z}^{n} .
$$

Then

$$
\begin{aligned}
& \sum_{n=2}^{\infty} \frac{\lambda_{n}((1+k) n-(k+\alpha))}{n(1-\alpha)}\left(\frac{n(1-\alpha)}{\lambda_{n}((1+k) n-(k+\alpha))}\right) X_{n} \\
& \quad+\sum_{n=1}^{\infty} \frac{\mu_{n}((1+k) n+(k+\alpha))}{n(1-\alpha)}\left(\frac{n(1-\alpha)}{\mu_{n}((1+k) n+(k+\alpha))}\right) Y_{n} \\
& =\sum_{n=2}^{\infty} X_{n}+\sum_{n=1}^{\infty} Y_{n}=1-X_{1} \leq 1,
\end{aligned}
$$

and so $f(z) \in \operatorname{clco} \overline{H S T}(\phi, \chi, k, \alpha)$. Conversely, suppose that $f(z) \in \operatorname{clco} \overline{H S T}(\phi, \chi, k, \alpha)$. Set

$$
X_{n}=\frac{\lambda_{n}((1+k) n-(k+\alpha))}{n(1-\alpha)}\left|a_{n}\right| \quad(n \geq 2)
$$

and

$$
Y_{n}=\frac{\mu_{n}((1+k) n+(k+\alpha))}{n(1-\alpha)}\left|b_{n}\right| \quad(n \geq 1),
$$

then note that by Theorem $2,0 \leq X_{n} \leq 1(n \geq 2)$ and $0 \leq Y_{n} \leq 1(n \geq 1)$.

Consequently, we obtain

$$
f(z)=\sum_{n=1}^{\infty}\left[X_{n} h_{n}(z)+Y_{n} g_{n}(z)\right]
$$

Using Theorem 2, it is easily seen that the class $\overline{H S T}(\phi, \chi, k, \alpha)$ is convex and closed and so $\operatorname{clco} \overline{H S T}(\phi, \chi, k, \alpha)=\overline{H S T}(\phi, \chi, k, \alpha)$.

\section{Convolution result}

For harmonic functions of the form

$$
f(z)=z-\sum_{n=2}^{\infty}\left|a_{n}\right| z^{n}+\sum_{n=1}^{\infty}\left|b_{n}\right| \bar{z}^{n}
$$

and

$$
G(z)=z-\sum_{n=2}^{\infty} A_{n} z^{n}+\sum_{n=1}^{\infty} B_{n} \bar{z}^{n} \quad\left(A_{n}, B_{n} \geq 0\right)
$$


we define the convolution of two harmonic functions $f$ and $G$ as

$$
(f * G)(z)=f(z) * G(z)=z-\sum_{n=2}^{\infty} a_{n} A_{n} z^{n}+\sum_{n=1}^{\infty} b_{n} B_{n} \bar{z}^{n} .
$$

Using this definition, we show that the class $\overline{H S T}(\phi, \chi, k, \alpha)$ is closed under convolution.

Theorem 5 For $0 \leq \alpha<1$, let $f \in \overline{H S T}(\phi, \chi, k, \alpha)$ and $G \in \overline{H S T}(\phi, \chi, k, \alpha)$. Then $f(z) *$ $G(z) \in \overline{H S T}(\phi, \chi, k, \alpha)$.

Proof Let the functions $f(z)$ defined by (4.1) be in the class $\overline{H S T}(\phi, \chi, k, \alpha)$, and let the functions $G(z)$ defined by (4.2) be in the class $\overline{H S T}(\phi, \chi, k, \alpha)$. Obviously, the coefficients of $f$ and $G$ must satisfy a condition similar to inequality (2.4). So, for the coefficients of $f(z) * G(z)$, we can write

$$
\begin{aligned}
& \sum_{n=2}^{\infty} \frac{\lambda_{n}}{n} \frac{(1+k) n-(k+\alpha)}{(1-\alpha)}\left|a_{n}\right| A_{n}+\sum_{n=1}^{\infty} \frac{\mu_{n}}{n} \frac{(1+k) n+(k+\alpha)}{(1-\alpha)}\left|b_{n}\right| B_{n} \\
& \quad \leq \sum_{n=2}^{\infty}\left[\frac{\lambda_{n}}{n} \frac{(1+k) n-(k+\alpha)}{(1-\alpha)}\left|a_{n}\right|+\sum_{n=1}^{\infty} \frac{\mu_{n}}{n} \frac{(1+k) n+(k+\alpha)}{(1-\alpha)}\left|b_{n}\right|\right],
\end{aligned}
$$

the right-hand side of this inequality is bounded by 1 because $f \in \overline{H S T}(\phi, \chi, k, \alpha)$. Then $f(z) * G(z) \in \overline{H S T}(\phi, \chi, k, \alpha)$.

Finally, we show that $\overline{H S T}(\phi, \chi, k, \alpha)$ is closed under convex combinations of its members.

Theorem 6 The class $\overline{H S T}(\phi, \chi, k, \alpha)$ is closed under convex linear combination.

Proof For $i=1,2,3, \ldots$, let $f_{i} \in \overline{H S T}(\phi, \chi, k, \alpha)$, where the functions $f_{i}$ are given by

$$
f_{i}(z)=z-\sum_{n=2}^{\infty}\left|a_{n, i}\right| z^{n}+\left.\sum_{n=1}^{\infty}\left|b_{n, i}\right|\right|^{n}
$$

For $\sum_{i=1}^{\infty} t_{i}=1 ; 0 \leq t_{i} \leq 1$, the convex linear combination of $f_{i}$ may be written as

$$
\sum_{i=1}^{\infty} t_{i} f_{i}(z)=z-\sum_{n=2}^{\infty}\left(\sum_{i=1}^{\infty} t_{i}\left|a_{n, i}\right|\right) z^{n}+\sum_{n=1}^{\infty}\left(\sum_{i=1}^{\infty} t_{i}\left|b_{n, i}\right|\right) \bar{z}^{n},
$$

then by (2.4) we have

$$
\begin{aligned}
& \sum_{n=2}^{\infty} \frac{\lambda_{n}}{n} \frac{(1+k) n-(k+\alpha)}{(1-\alpha)} \sum_{i=1}^{\infty} t_{i}\left|a_{n, i}\right|+\sum_{n=1}^{\infty} \frac{\mu_{n}}{n} \frac{(1+k) n+(k+\alpha)}{(1-\alpha)} \sum_{i=1}^{\infty} t_{i}\left|b_{n, i}\right| \\
& \quad=\sum_{i=1}^{\infty} t_{i}\left\{\sum_{n=2}^{\infty}\left[\frac{\lambda_{n}}{n} \frac{(1+k) n-(k+\alpha)}{(1-\alpha)}\left|a_{n, i}\right|+\sum_{n=1}^{\infty} \frac{\mu_{n}}{n} \frac{(1+k) n+(k+\alpha)}{(1-\alpha)}\left|b_{n, i}\right|\right]\right\} \\
& \quad \leq \sum_{i=1}^{\infty} t_{i}=1 .
\end{aligned}
$$


This condition is required by (2.4) and so $\sum_{i=1}^{\infty} t_{i} f_{i}(z) \in \overline{H S T}(\phi, \chi, k, \alpha)$. This completes the proof of Theorem 6 .

\section{Remarks}

(i) Putting $k=0$ in our results, we obtain the results obtained by Dixit et al. [9];

(ii) Putting $\varphi(z)=\chi(z)=\frac{z}{(1-z)^{2}}$ and $k=1$ in our results, we obtain the results obtained by Rosy et al. [10];

(iii) Putting $\varphi(z)=\chi(z)=\frac{z+z^{2}}{(1-z)^{3}}$ in our results, we obtain the results obtained by Kim et al. [8];

(iv) Putting $\varphi(z)=\chi(z)=\frac{z}{(1-z)^{2}}$ and $k=0$ in our results, we obtain the results obtained by Jahangiri [3];

(v) Putting $\varphi(z)=\chi(z)=\frac{z+z^{2}}{(1-z)^{3}}$ and $k=0$ in our results, we obtain the results obtained by Jahangiri [2].

\section{Competing interests}

The author declares that they have no competing interests.

\section{Acknowledgements}

The author would like to express her sincere gratitude to Springer Open Accounts Team for their kind help.

Received: 6 December 2012 Accepted: 14 October 2013 Published: 12 Nov 2013

\section{References}

1. Clunie, J, Sheil-Small, T: Harmonic univalent functions. Ann. Acad. Sci. Fenn., Ser. A 1 Math. 9, 3-25 (1984)

2. Jahangiri, JM: Coefficient bounds and univalent criteria for harmonic functions with negative coefficients. Ann. Univ. Marie-Curie Sklodowska Sect. A 52, 57-66 (1998)

3. Jahangiri, JM: Harmonic functions starlike in the unit disc. J. Math. Anal. Appl. 235, 470-477 (1999)

4. Silverman, H: Harmonic univalent function with negative coefficients. J. Math. Anal. Appl. 220, 283-289 (1998)

5. Silverman, H, Silvia, EM: Subclasses of harmonic univalent functions. N.Z. J. Math. 28, 275-284 (1999)

6. Kanas, S, Wisniowska, A: Conic regions and k-uniform convexity. J. Comput. Appl. Math. 105, 327-336 (1999)

7. Kanas, S, Srivastava, HM: Linear operators associated with k-uniformly convex functions. Integral Transforms Spec. Funct. 9(2), 121-132 (2000)

8. Kim, YC, Jahangiri, JM, Choi, JH: Certain convex harmonic functions. Int. J. Math. Math. Sci. 29(8), 459-465 (2002)

9. Dixit, KK, Pathak, AL, Porwal, S, Agarwal, R: On a subclass of harmonic univalent functions defied by convolution and integral convolution. Int. J. Pure Appl. Math. 69(3), 255-264 (2011)

10. Rosy, T, Stephen, BA, Subramanian, KG, Jahangiri, JM: Goodman-Ronning-type harmonic univalent functions. Kyungpook Math. J. 41, 45-54 (2001)

11. Joshi, SB, Darus, M: Unified treatment for harmonic univalent functions. Tamsui Oxford Univ. J. Math. Sci. 24(3), 225-232(2008)

10.1186/1029-242X-2013-537

Cite this article as: El-Ashwah: Subclass of univalent harmonic functions defined by dual convolution. Journal of Inequalities and Applications 2013, 2013:537

\section{Submit your manuscript to a SpringerOpen ${ }^{\circ}$ journal and benefit from:}

- Convenient online submission

- Rigorous peer review

- Immediate publication on acceptance

Open access: articles freely available online

- High visibility within the field

- Retaining the copyright to your article 